signalling could be potential therapies for the prevention of vascular calcification.

\section{THE IMPACT OF NADPH OXIDASE 2 INHIBITION ON SKELETAL MUSCLE PATHOPHYSIOLOGY OF ATHEROSCLEROTIC MICE}

\begin{abstract}
${ }^{1}$ Pagona Sfyri ${ }^{*},{ }^{2}$ Nadira Y. Yuldasheva, ${ }^{3}$ Anastasia Tzimou, ${ }^{3}$ Vassilis Mougios, ${ }^{2}$ Mark Kearney, ${ }^{1}$ Antonios Matsakas. 'Molecular Physiology Laboratory, Centre for Atherothrombotic and Metabolic Disease, Hull York Medical School; ' ${ }^{2}$ Leeds Institute of Genetics Health and Therapeutics, University of Leeds; ${ }^{3}$ School of Physical Education and Sports Science, Aristotle University of Thessaloniki
\end{abstract}

\subsection{6/heartjnl-2017-311726.224}

Introduction Apolipoprotein E-deficient mice $\left(\mathrm{ApoE}^{-/}\right)$develop severe hyperlipidaemia and atherosclerotic lesions throughout the aortic root, features that are aggravated by atherogenic diets. NADPH oxidase 2 (Nox2) is an important enzymatic source of reactive oxygen species that contributes to systemic atherosclerosis in $\mathrm{ApoE}^{-/-}$mice. The aim of this study was to investigate the role of Nox 2 inhibition in skeletal muscle pathophysiology and cellular oxidative stress of $\mathrm{ApoE}^{-/-}$mice administered a Western-type of diet (WD).

Methods $\mathrm{ApoE}^{-/-}$mice were maintained on either a chow or a Western diet for 12 weeks and were treated with the Nox2dstat inhibitor or control peptide for the last 8 weeks of feeding. Skeletal muscles and the liver were dissected for molecular, biochemical and histological analysis.

Results Individual muscle fibres from $\mathrm{ApoE}^{-/-}$mice were significantly enlarged due to ectopic fat accumulation. There was an increase in hepatic inflammation and lipid deposition in response to WD administration. Importantly, there was perturbed gene expression for fatty acid metabolism and antioxidant genes, followed by evidence of oxidative stress, as shown by elevated lipid peroxidation and oxidative protein modifications such as carbonylation and tyrosine nitration in the skeletal muscle of WD-fed mice. Pharmacological inhibition of Nox2 decreased superoxide production and protein carbonylation, one of the most harmful protein modifications, in the muscle of $\mathrm{ApoE}^{-/-}$mice but had no effect on the liver.

Conclusions Our data indicate that ApoE deficiency induces oxidative damage in skeletal muscle and hepatic steatosis that are more profound under Western diet. Nox2 inhibition attenuates oxidative stress in skeletal muscle and holds promise for counteracting the impact of peripheral atherosclerosis in skeletal muscle. This study provides key evidence to better understand the pathophysiology of skeletal muscle in peripheral atherosclerosis and arterial disease; it also identifies alternative therapies to combat muscle oxidative stress.

\section{INFARCT SIZE IN A RAT MODEL OF ACUTE MYOCARDIAL INFARCTION IS REDUCED BY INTERLEUKIN-6 TRANS-SIGNALLING BLOCKADE USING SGP130FC BUT NOT AN ANTI-IL-6R MONOCLONAL ANTIBODY}

Marc Jonathan George*, Daniel Stuckey, Valerie Taylor, Aroon Hingorani, Derek Gilroy. University College London

\subsection{6/heartjnl-2017-311726.225}

Introduction Interleukin-6 (IL-6) is elevated during acute myocardial infarction (AMI) particularly after reperfusion with primary percutaneous coronary intervention (PPCI). Higher circulating levels of IL-6 and its soluble receptor (sIL-6R) are associated with adverse outcomes post AMI. Therefore while IL-6 is a potential therapeutic target in AMI, animal models employing monoclonal antibodies (MAb) against the IL-6R have failed to demonstrate benefit. We hypothesised that blockade of the pro-inflammatory aspects of IL-6 signalling (trans-signalling) with the sgp $130 \mathrm{Fc}$ protein in an animal model of AMI would result in reduced infarct size (IS) whereas blockade with a MAb against IL-6R (which blocks both the pro and anti-inflammatory actions of IL-6) would not.

Methods AMI was induced in male Sprague-Dawley rats by occluding the left-anterior descending artery for 50 minutes prior to reperfusion (analogous to PPCI). The model was characterised by measuring the temporal profile of IL-6, sIL$6 \mathrm{R}$ and other inflammatory mediators (MCP-1, KC/GRO, IL$1 \beta, \mathrm{TNF} \alpha$ ) within the heart tissue and plasma by ELISA at 2, 4, 24, 72, 120 and 168 hours post AMI (n=3-4/group). In addition, infarct progression over time (measured histologically with TTC and Evans Blue dyes and with plasma myoglobin), and leukocyte infiltration (flow-cytometry of cells obtained from heart digests) were measured. In therapeutic experiments rats received either $4 \mu \mathrm{g} / \mathrm{g}$ of a MAb against IL-6R (clone 15A7), $0.5 \mu \mathrm{g} / \mathrm{g}$ of $\mathrm{sgp} 130 \mathrm{Fc}$ or vehicle alone given intravenously 1 minute prior to reperfusion ( $n=7-8 /$ group).

Results IS/Area at risk (AAR) increased from $31.81 \%$ at 4 hours to $46.1 \%$ at 24 hours $(p=0.03)$, with no further change at 48 hours. Myoglobin peaked at 24 hours. IL-6 levels in the heart were biphasic; a robust early peak at 24 hours was followed by a trough at 24 hours, and a more sustained peak between days 3-5. Only the early peak was associated with significantly elevated circulating IL-6. The early peak was temporally associated with infarct progression and neutrophil influx, whereas the second was associated with classical mononcyte infiltration. Other inflammatory mediators followed a similar but less pronounced biphasic pattern. Cardiac and plasma sIL-6R peaked at 24 hours, coinciding with maximal cardiac neutrophil numbers. Based on these data the effect of IL-6 antagonism was assessed at 24 hours. IS/AAR after blockade with anti-IL-6R MAb was unchanged compared with control $(46.8 \%$ vs $46.1 \%)$. However, blockade with sgp130Fc resulted in a substantial reduction in IS/ARR (26.32\%, p 0.0004).

Conclusions IL-6 trans-signalling blockade with sgp130Fc but not blockade with an anti-IL-6R MAb reduces IS/AAR in an animal model of AMI with reperfusion. Ongoing experiments seek to understand the mechanisms underpinning this observation and to explore the effects on infarct healing and remodelling.

\section{BRAF INHIBITORS, SB590885 AND DABRAFENIB, ENHANCE ERK1/2 SIGNALLING IN CARDIOMYOCYTES AND PROMOTE CARDIAC HYPERTROPHY}

Daniel Meijles*, Michelle Hardyman, Kerry Rostron, Stephen Fuller, Peter Sugden, Angela Clerk. University of Reading

\subsection{6/heartjnl-2017-311726.226}

Purpose ERK1/2 are phosphorylated and activated by MKK1/2 that are phosphorylated and activated by Raf kinases. ERK1/2 promote cardiomyocyte hypertrophy and is protective, whereas 
they promote proliferation in cancer cells. Mutations in BRaf are commonly found in cancer, and drugs targeting the enzyme are in clinical use. Of these, the type I inhibitor, SB590885 (SB), binds to the active conformation of BRaf, whilst dabrafenib has no activation state selectivity. Paradoxically, these inhibitors activate ERK1/2 in cancer cells by stimulating the cascade at low concentrations rather than inhibiting it. Our aim is to determine the effects of BRaf inhibitors on the ERK1/2 cascade in cardiomyocytes to establish whether they may activate the pathway in the hearts of patients undergoing cancer therapy.

Methods Rat neonatal cardiomyocytes were exposed to dabrafenib or $\mathrm{SB}$ and the effects on activation of $\mathrm{MKK} 1 / 2$ or ERK1/2 examined by immunoblotting with antibodies to phosphorylated (i.e. activated) or total kinases. Raf activities were assayed using GST-MKK1 as a substrate. The effects on myocyte morphology were studied by immunostaining for myofibrillar proteins.

Results Low concentrations of SB $(0.1 \mu \mathrm{M})$ or dabrafenib (1 $\mu \mathrm{M}$ ) enhanced ERK1/2 signalling, with SB having a greater effect than dabrafenib. Thus, cardiomyocytes are primed for paradoxical activation of ERK1/2 by BRaf inhibitors. One explanation for the Raf paradox is that BRaf forms homo or heterodimers with cRaf. Thus, a submaximal concentration of BRaf inhibitor may be sufficient to inhibit only one Raf protein and, since the kinase is locked in an active state, there is activation of the other Raf partner. Consistent with this, 0.1 $\mu \mathrm{M}$ SB increased activities of both BRaf and cRaf, although there was no significant increase in dimerization. High concentrations of SB or dabrafenib $(1-10 \mu \mathrm{M})$ inhibited basal ERK1/ 2 activity in cardiomyocytes, but the inhibitory effect was only transient and levels returned to basal within 1 hour. Furthermore, high concentrations of SB activated ERK1/2 over prolonged periods (up to 24 hour). This was associated with an increase in cardiomyocyte size and myofibrillar organisation consistent with low level activation of the pathway promoting cardiomyocyte hypertrophy. In mouse hearts in vivo, dabrafenib and SB590885 enhanced MKK1/2 phosphorylation over 24 hour, and increased hypertrophic ANF and BNP mRNA expression markers, consistent with the cell data.

Conclusions Cardiomyocytes are primed for Raf paradox signalling and BRaf inhibitors such as SB and dabrafenib activate ERK1/2 in cardiomyocytes. Consistent with a role for ERK1/2 in cardiomyocyte hypertrophy, SB increases expression of hypertrophic gene markers and promotes morphological changes associated with hypertrophy.

\section{ROLE OF SUMOYLATION AND DESUMOYLATION OF MITOCHONDRIAL FISSION PROTEINS IN MYOCARDIAL ISCHAEMIA-REPERFUSION INJURY}

${ }^{1}$ Nadiia Rawlings*, ${ }^{1}$ Laura Lee, ${ }^{2}$ Jordan Martin, ${ }^{1}$ Richard Seager, ${ }^{3}$ Chun Guo, ${ }^{1}$ Kevin Wilkinson, ${ }^{1}$ Andrew Halestrap, ${ }^{1}$ Jeremy Henley. ' University of Bristol; ${ }^{2}$ University of Plymouth; ${ }^{3}$ University of Sheffield

\subsection{6/heartjnl-2017-311726.227}

Background Restoration of blood supply to the heart after a prolonged ischaemic episode causes myocardial ischaemiareperfusion (I/R) injury. Paradoxically, short periods of ischaemia and reperfusion, known as preconditioning, has a potent cardioprotective effect. One key aspect of $\mathrm{I} / \mathrm{R}$ injury is cytotoxic mitochondrial fission that allows cytochrome $c$ release and promotes apoptotic cell death. Dynamin-related protein 1
(Drp1) is a large GTPase that is recruited from the cytosol to the mitochondrial outer membrane to drive fission. Drp1 function is regulated by posttranslational modifications, including conjugation to small ubiquitin-like modifier (SUMO)-2/3. SUMO-2/3-ylation decreases Drp1 partitioning to the mitochondrial membrane, which reduces stress-induced fission and apoptosis. We have shown previously that, in neurons, Drp1 SUMO-2/3-ylation is controlled by the deSUMOylating enzyme SENP3, levels of which are reduced during ischaemia but restored upon reperfusion.

Objective Here we investigated alterations in SUMOylation and Drp1 partitioning in heart during ischaemia, I/R and preconditioning. Methods: Isolated hearts from male Wistar rats were perfused using Langendorff apparatus with Krebs Henseleit solution. Hearts were randomly divided into 4 groups (with $\mathrm{n}=5-6$ per group): control (50 min perfusion); ischaemia (20 min perfusion +30 min ischaemia); preconditioning (3 short cycles of I/R, 2 and 3 mins, respectively, followed by $30 \mathrm{~min}$ of ischaemia); $I / R$ (30 min of ischaemia followed by 2 hour of reperfusion). All samples were immediately subjected to subcellular fractionation, then frozen on dry ice and used for Western blot analysis.

$1 \mathrm{~A}$

Total SENP3.

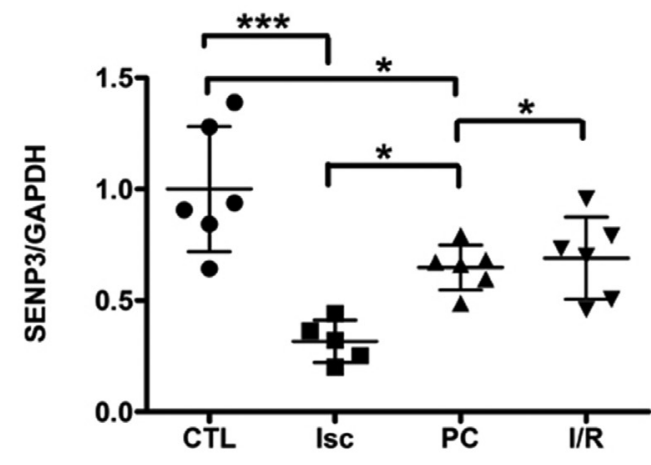

1B

Total SUMO-2/3-ylation. Mitochondrial fraction.

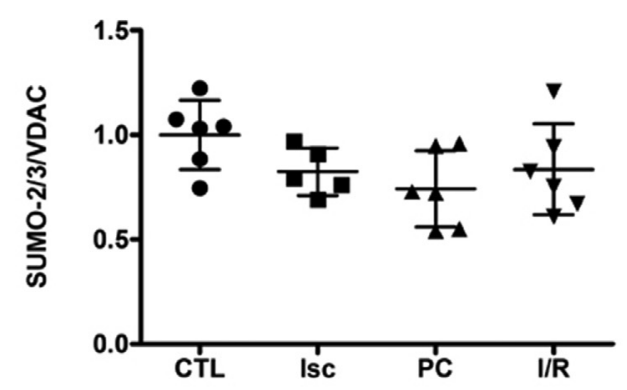

Abstract 229 Figure 1

Results Levels of SENP3 were reduced in all three groups compared to control, with the greatest reduction seen during ischaemia (Fig 1A). Despite the observed reduction in SENP3 levels no obvious change in overall levels of protein SUMO-2/ 3 -ylation was detected in mitochondria fractions (Fig 1B). An increase of SUMO-1 conjugation to an unidentified protein at about $55 \mathrm{kDa}$ was observed during ischaemia and $\mathrm{I} / \mathrm{R}$ 